\title{
Second-Order Statistical Methods GLCM for Authentication Systems
}

\author{
Mohammed A. Taha*, Hanaa M. Ahmed \\ Computer Science Department, University of Technology, Baghdad, Iraq,
}

Correspondence

*Mohammed A. Taha

Computer Science Department,

University of Technology, Baghdad, Iraq

Email: cs.19.54@grad.uotechnology.edu.iq

\begin{abstract}
For many uses, biometric systems have gained considerable attention. Iris identification was One of the most powerful sophisticated biometrical techniques for effective and confident authentication. The current iris identification system offers accurate and reliable results based on near-infrared light (NIR) images when images are taken in a restricted area with fixeddistance user cooperation. However, for the color eye images obtained under visible wavelength $(V W)$ without collaboration among the users, the efficiency of iris recognition degrades because of noise such as eye blurring images, eye lashing, occlusion, and reflection. This work aims to use the Gray-Level Co-occurrence Matrix (GLCM) to retrieve the iris's characteristics in both NIR iris images and visible spectrum. GLCM is second-order Statistical-Based Methods for Texture Analysis. The GLCMbased extraction technology was applied after the preprocessing method to extract the pure iris region's characteristics. The Energy, Entropy, Correlation, Homogeneity, and Contrast collection of second-order statistical features are determined from the generated co-occurrence matrix, Stored as a vector for numerical features. This approach is used and evaluated on the CASIA vland ITTD $v 1$ databases as NIR iris image and UBIRIS $v 1$ as a color image. The results showed a high accuracy rate (99.2 \%) on CASIA v1, (99.4) on ITTD v1, and (87\%) on UBIRIS v1 evaluated by comparing to the other methods.
\end{abstract}

KEYWORDS: Biometrics system, Feature extraction, GLCM, Iris recognition.

\section{INTRODUCTION}

Iris recognition (IR) is now a common biometric technique used to identify people based solely on the iris texture. Iris contains more robust and special features among a variety of biometric traits. Iris has received comprehensive applications, such as the Aadhar Program in India, Amsterdam Airport, the United States / Canadian Borders, etc. Studies have studied excellent developments in iris detection over the last decade, and some existing approaches have demonstrated up to $99 \%$ accuracy[1]. However, IR is faced with several challenges, including unregulated image acquisition. Iris acquisition is typically performed in a complex environment[2] in which an individual focuses on a distance on the camera/sensor during the captioning of images. Uncontrolled photographs with consistent image quality are not desirable.

Certain portions of the iris are not captured when the eye is opened partly or defocused[3]. The usefulness of a broad range of current algorithms also depends on the precise segmentation of the iris. However, the surrounding variables, such as the eyelashes and eyelids, cover a broad iris region, affecting segmentation and recognition. In addition, iris images also withstand occlusion, camera diffusion, distortion, variation, in Contrast, movement blur, pupil dilation, and luminosity, etc. [4]. Fig1 displays several pictures of the iris sample, such as the above issues.

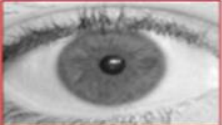

(a)

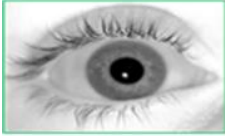

(e)

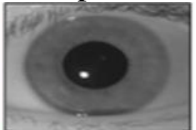

(b)

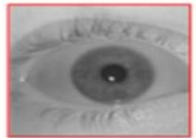

(f)

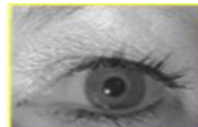

(c)

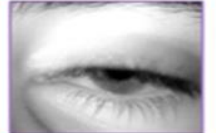

(g)

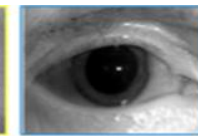

(d)

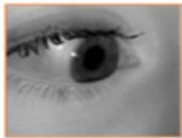

(h)
Fig. 1: Unconstrained iris images (a) reflection, (b) motion blur, (c) contrast variation, (d) pupil dilation, (e )occlusion, (f) blurred image, (g) partially open eye and (h) defocused image[1]

Feature extraction is a significant attempt to obtain essential characteristics from iris objects. Feature extraction authors have adopted various algorithms based on different transformations[5]. A few studies have been documented using iris recognition statistical techniques. Therefore, this paper proposes and implements a second-order statistical extraction method based on GLCM to extract appropriate iris texture features[6][7][8]. For a single direction and to 
combine all the orientations together to make GLCM independent, GLCM features can be obtained. It's a metric for the various combinations of pixel brightness values that can appear in an image. System efficiency is measured to achieve an accuracy rate, false acceptance rate (FAR), false refusal rate (FRR), and a genuine acceptance rate (GAR). There are many ways to retrieve the properties of the iris captured by NIR, but there are a few ways to retrieve the properties of the iris in VW and NIR.

The wavelength of NIR is particularly dangerous because its natural mechanisms do not instinctively react to the eye (pupil contraction, blinking, and aversion). Using visible light and unregulated imaging setups may reduce the data quality, making it challenging to identify reliable recognition. So, this paper proposes to use the GLCM method to retrieve properties in the NIR iris images and visible wavelength (VW) iris images.

This paper is structured as follows. Section 2 gives a concise description of the proposed Iris recognition system and explains all its steps. In Section 3, results are presented and compared with existing technologies. Finally, section 4 concludes the paper.

\section{Proposed Methology}

One of the most comprehensive human identification schemes is the Iris Recognition System. In Contrast to other biometric technologies, Iris detection is a reasonably recent biometric method. Compared to other biometric systems, such as the face, fingerprint, speech retina, etc. Fig 2 displays the iris recognition system schematic diagram. The extraction of the Iris attribute is used to extract the most discriminating characteristic of an iris image [9]. It is a specific type of dimension reduction and provides the most information about an iris's actual image. The feature coefficient is encoded after the feature is extracted to make a convenient and accurate comparison between templates. This study focuses on extraction iris texture features taken from the database CASIA v1 and ITTD v1.

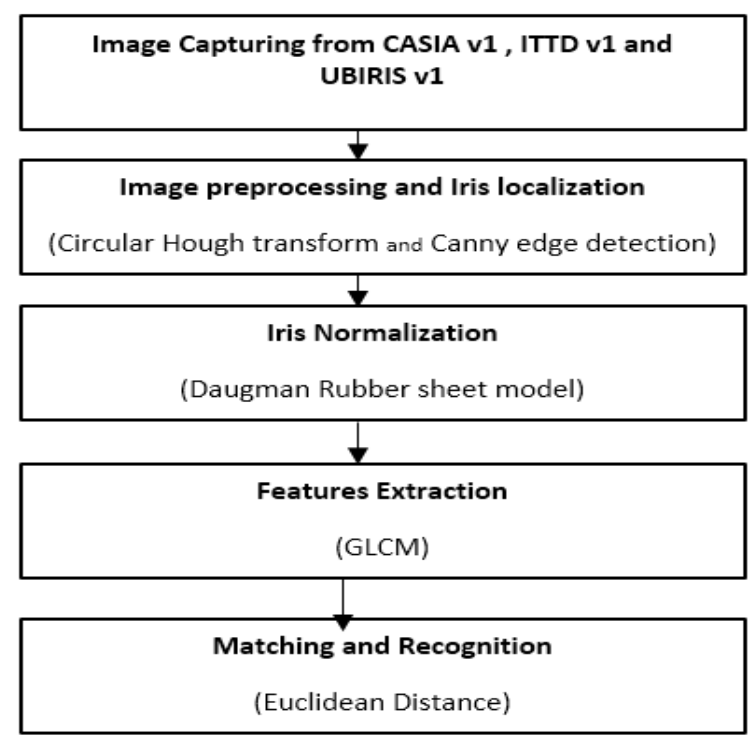

Fig. 2: Iris recognition systems.

\section{1) Image acquisition}

The first stage consists of acquiring an input image from various devices. The quality of the image affects the overall performance of the recognition system. In this work, the collection of images from CASIA v1 and ITTD v1[3]. CASIA v1 consists of 756 iris images of 108 persons and seven images each taken during two separate sessions with a difference of at least one month. Both 8-bit grey images are 320 to 280 resolution and preserved in bitmap format.

The IITD v1 image database mainly consists of iris pictures obtained from IIT Delhi students and staff. This database was compiled by JIRIS, JPC1000, and digital CMOS camera in the Biometrics Research Laboratory from January to July 2007. The collected images have been stored in bitmap format. The database includes 2,240 images from 224 different users that are freely available to researchers. In the sample, all subjects aged 14-55 years consist of 176 men and 48 women. These pictures are $320 \times 240$ pixels in size, and all these photographs have been acquired indoors.

UBIRIS.v1 contains 1877 photos obtained from 241 people in two separate sessions in September 2004. The most significant aspect is the integration of images with multiple noise factors, which simulate a less restricted imaging environment. The robustness of iris reconnaissance methods can be measured. For the first capture image session, which is the enrollment phase, we tried, in particular, to minimize the noise factors relating to reflections, illumination, and Contrast. We modified the position of capture in the second session to add natural luminosity. This enables heterogeneous images to appear in relation to reflections, Contrast, light, and difficulties with concentrating[10]. CASIA v1 and ITTD v1 are NIR imaging datasets, while ITTD $v 1$ are VW noisy image datasets.

\section{2) Image Preprocessing and Iris localization}

After the eye image has been captured or loaded, the next step is preprocessing. Images are pre-processed to enhance the machine's ability to identify characteristics and artifacts. Preprocessing can be as easy as adapting intensity, including stretching intensity, histogram equalization, noise reduction. The iris is taken using a high-resolution camera. To delete irrelevant objects, such as the eyelash, pupil, etc., the original image must be preprocessed. Pre-processing images is a critical step in computer vision applications to eliminate the image noise and prepare the iris image for a bit of noise.

Iris location is among the essential steps in the iris recognition scheme. The inner and outer limits of the iris are located. A sclera, iris, and pupil. Colored white and out of iris, Sclera. The pupil is in the iris, and its size differs due to the strength of the light. Iris contains information about texture, so it must be the iris and the pupil. In [11], there is an algorithm to locate the eye with a sequence of steps that can yield good results by using this method with Circular Hough Transformation (CHT)[12]. The segmentation is based on the circular Hugh Transform to detect the iris field, limited to a manually-set interval, depending on the database used[13]. The Hough Transforms and Canny Edge detection line isolates the eyelids[14]. A threshold excludes the eyelids and possible reflections, as seen in Fig 3. 


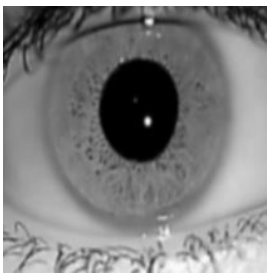

(a) ITTD original image

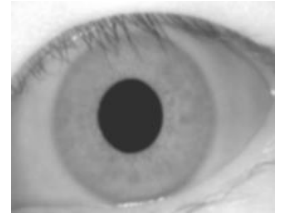

(c) CASIA original image

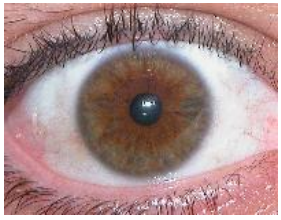

(e) UBIRIS original image

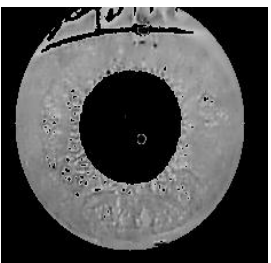

(b) ITTD cropped iris

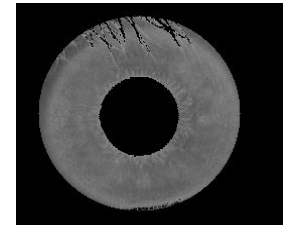

(d) CASIA cropped iris

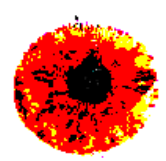

(f) UBIRIS cropped Iris

Fig. 3: Original and Cropped Iris from CASIA v1, ITTD v1, and UBIRIS v1 Database.

\section{3) Iris Normalization}

Normalization involves the conversion from Polar to Cartesian Coordinates of the object. If the iris image has successfully been found, The next step is to turn the iris region into fixed dimensions. However, there are many algorithms available in this work, Daugman Rubber Sheet Model, as used[15]. Figure 4 shows the model rubber sheet, which restores the points in the iris area to a pair of polar coordinates $(r, \theta)$ with $r$ at the interval $[0,1]$ and $\theta[0,2 \mu]$ at the angles.

The image of the iris, with its fixed size, helps the extraction technique to compare the two pictures of the iris[11][15]. Due to the dilation of the pupil from changing illumination levels, dimensional differences may occur. Otherwise, dimensional incoherence can be induced within an eye socket by varying image distance, head tilt, camera rotation, and eye rotation. This means that the normalization process is necessary to provide two images of the same iris under different conditions.

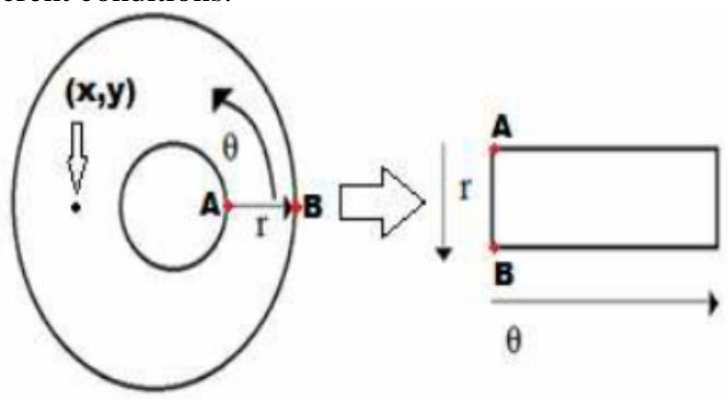

Fig. 4: Daugman's rubber sheet model.

\section{4) Iris Features Extraction by GLCM}

The features that result from the statistics in the first order provide information on the gray-level image distribution but do not include any information on the relative positions of the various grays within the image. The second-order statistics are used to provide this detail, where the pixels are taken into account in pairs. Statistics from the second and higher order predict two or more pixel values of each other at particular locations. Gray Level Co-occurrence Matrices are examples of static texture second-order characteristics. Recently, one of the specialized techniques used to obtain texture features is the Gray Level Co-occurrence Matrix (GLCM) that has recently been proposed by Haralick in 1973[6][16]. It specifies the frequency with which a pixel value is known as the point of interest or (reference pixel) with the intensity value I, for a pixel value, in particular, called the next pixel with the intensity value. Therefore, each element $(\mathrm{I}, \mathrm{J})$ of the matrix represents the number of pairs with the value $I$ and a pixel with value $J$ at a distance or offsets $\mathrm{d}$ from one another. The relation between different adjacent pixels in four possible spatial relations with different offsets and angles can be described in several ways. $(0 ; 45 ; 90$ and 135) as seen in Fig 5.
a)
b)

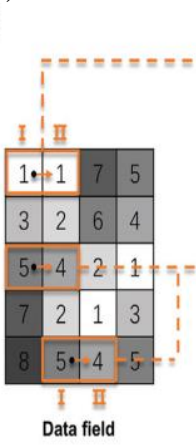

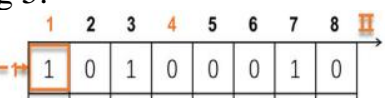

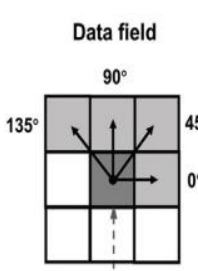 Point of interest \begin{tabular}{llllllllll|}
\hline 2 & 2 & 0 & 0 & 0 & 0 & 1 & 0 & 0 \\
\hline
\end{tabular} \begin{tabular}{lllllllllll}
3 & 0 & 1 & 0 & 0 & 0 & 0 & 0 & 0 \\
\hline
\end{tabular}

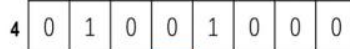 $-5=0=\theta=\theta=2$\begin{tabular}{lllllll}
-0 & 0 & 0 & 0 & 0 \\
\hline
\end{tabular}

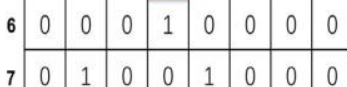 \begin{tabular}{|l|l|l|l|l|l|l|l|}
\hline 0 & 0 & 0 & 0 & 1 & 0 & 0 & 0 \\
\hline
\end{tabular} GLCM matrix

Fig. 5: (a) Matrix directions for obtaining texture characteristics. (b) The eight gray levels and $0^{\circ}$ direction are used as an illustration in this diagram of the co-occurrence matrix formation. Set up a couple's two gray values as a coordinate (I, II) in the appropriate locations of the cooccurrence matrix to every statistic. The number " 2 " in row 5 and column 4 of the co-occurrence matrix, for example, means that there are two couples of gray-level 5 that are adjacent to gray-level 2 along the $0^{\circ}$ direction[17] .

A large number of texture features can be derived from the GLCM, which can be described as following [6][14][7]:

- Contrast: Evaluates the local gray level variation in pixel intensity values. The Contrast is insufficient if the gray concentrations of each pair of pixels are comparable, as given by:

$$
\text { Contrast }=\sum_{i} \sum_{j}(i-j)^{2} N_{d}(i, j)
$$

- Where $I \mathrm{j}$, the number of lines and columns in the gray matrix, and $\mathrm{Nd}(\mathrm{i}, \mathrm{j})$, the probability that the pixel intensity values are found in an image. Contrast is poor if all of the next pixels have the same intensity values. The gray level variation itself reflects the texture variation.

- Energy: The count of repeated pairs is calculated. The energy should be high if repeated pixel pairs are high.

$$
\text { Energy }=\sum_{i} \sum_{j} N_{d}^{2}(i, j)
$$

- Entropy: Describes the amount of randomness necessary for image compression. It is given by:

$$
\text { Entropy }=-\sum_{i} \sum_{j} N_{d}(i, j) \log _{2} N_{d}(i, j)
$$


- Homogeneity: Tests the pixel pair's local homogeneity. The homogeneity is supposed to be high if the pixel values of each pair of pixels are identical, as given by:

$$
\text { Homogenetiy }=\sum_{i} \sum_{j} \frac{N_{d}(i, j)}{1+|i-j|}
$$

- Correlation: Provides a connection in the pixel pair between both the two pixels. The correlation is supposed to be vital if the pixel pair gray levels are strongly correlated.

$$
\text { Correlation }=\frac{\sum_{i} \sum_{j}\left(1-\mu_{i}\right)\left(j-\mu_{j}\right) N_{d}(i, j)}{\sigma_{i} \sigma_{j}}
$$

Where $\mu_{i}, \mu_{j}$ Are the means and $\sigma_{i} \sigma_{j}$ Are the standard deviation of the row and column.

\section{5) Matching and Recognition}

In the function encoding process, the template generated also needs an appropriate Matching metric that provides a similarity measurement for the two iris models. This metric should include a range of values compared to the intra-Class comparisons and templates generated with the same eye, as well as another range of values in comparison of templates shaped by various irises. These two cases should have distinct values so that decisions about whether two models come from a single iris or two different irises can be determined with complete confidence. After proper extraction of the characteristic vectors, a similarity measurement is now compared. For making the right decision, Euclidean Distance (ED) is used. It is the most common distance used. The Euclidean distance calculates the square root of the sum of the vector squares. The ED is determined between the vector of the test iris (VT) and the defined vector (VC) of equation (6).

$$
E D=\sqrt{\sum(V T-V C)^{2}}
$$

\section{RESULTS AND DISCUSSION}

Three tests were used to determine the efficiency of our system: False Refusal Rate (FRR), False Acceptability Rate (FAR), and Genuine Accepted Rate (GAR). FAR represent false-accepted impostor attempts, while (FRR) represents genuine wrongly refused attempts. The genuine acceptance rate (GAR) is described as a percentage of valid system users, given as $100 \%$ (100-FRR); it is also known as recognition accuracy.

Experiments in two databases, IITD v1, CASIA v1, and UBIRIS v1, were performed in this work. The machine tested eye images for 100 individuals. Every individual has five photos, one for testing and the other for training. Figure 6 displays the number of iris images successfully segmented in both databases.

The results of the proposed iris encryption algorithm are shown in Fig. 6. The GAR of the proposed system is $(99.4 \%)$ for CASIA.v1, (99.7\%) for ITTD.v1 and (87.0\%) for UBIRIS v1. The images in the UBIRIS.v1 data set have been degraded by many aspects and are highly heterogeneous in terms of the lighting conditions in the environment. There is some degradation in the GAR because of in some cases, features could not be reliably extracted from a relatively large region in the iris pattern due to factors like occlusion reflection, motion blur, contrast variation, pupil dilation, blurred image, partially open eye, and defocused image. These images are the result of less constrained imaging conditions in various lighting conditions. This problem can be compensated by overlooks the occluded regions in the Euclidean distance calculation. However, this problem cannot be handled effectively by the proposed system leading to further false rejections. All datasets CASIA v1, UBIRIS v1, and ITTD.v1 have $(0.0 \%)$ FAR, making them suitable for a high-security application.

\section{EVAULATION RESULTS}

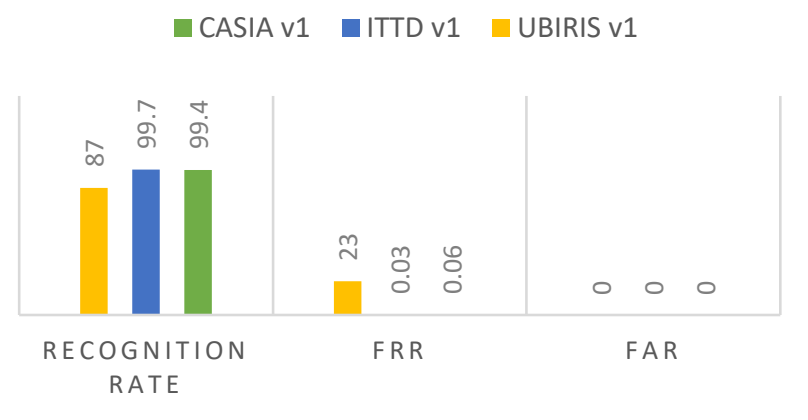

Fig. 6: Result analysis of different databases.

\section{COMPARISON With EXISTINg Algorithm}

The performance analysis of the Iris identification system depends on the FAR, FRR, ERR, rates of detection, and the number of coefficients required for the iris templates. This method has been compared to other current iris recognition systems. Table 1 demonstrates the Contrast of the detection rate and method of different algorithms.

Table 1.

Various algorithms and rates of recognition

\begin{tabular}{|l|l|l|}
\hline Authors & $\begin{array}{l}\text { Features } \\
\text { Extraction } \\
\text { Methods }\end{array}$ & $\begin{array}{l}\text { Recogniti } \\
\text { on rate }\end{array}$ \\
\hline J.Daugman[18] & Gabor filter & $100 \%$ \\
\hline $\begin{array}{l}\text { K. Gulmire and S. } \\
\text { Ganorkar[19] }\end{array}$ & Gabor wavelet & $99 \%$ \\
\hline $\begin{array}{l}\text { S.Hariprasd and } \\
\text { S.Venkatsubramian[20] }\end{array}$ & $\begin{array}{l}\text { Wavelet } \\
\text { packet }\end{array}$ & $93 \%$ \\
\hline Amir and Hamid[21] & $\begin{array}{l}\text { Contourlet } \\
\text { transform }\end{array}$ & $94.2 \%$ \\
\hline Tze Wang[22] & Haar wavelet & $98.45 \%$ \\
\hline Chia Te Chu[23] & LPCC & $96.8 \%$ \\
\hline V.Velisaulesevic[24] & Directionlets & $97.4 \%$ \\
\hline $\begin{array}{l}\text { M. Z. Rashad and } \\
\text { etc[25]. }\end{array}$ & LBP & $99.87 \%$ \\
\hline Proposed methods & GLCM & $99.4 \%$ \\
\hline
\end{tabular}

Table 1 shows that the Daugman method has higher rates of recognition than all other current algorithms. It provides a recognition rate of 100 percent. But the approach of Daugman is usually slower than any different current algorithm. It used several bits to compare two iris templates. GLCM gives low discrimination accuracy but a faster time when compared to Daugman's method. Both the GLCM and 
the Local Binary (LBP) are second-order statistical techniques for extracting the features of the iris texture, but the main weaknesses of the LBP technique are the "computing cost," which uses unworkable implementation for the calculation of pixels by pixels. LBP feature computation is a time-consuming process.

\section{CONCLUSIONS}

In this work, a statistical calculation of the second-order was based on the GLCM procedure to extract iris. The methodology for this paper texture extracting functionality and values are added certain features have been measured accordingly. This study shows it's possible to perform accurate biometric recognition using both VW data collected under complex lighting conditions and with no constraints and the use of NIR in image capturing in high constrains conditions environments. By evaluating the proposed system, we conclude that the iris recognition systems give better results by obtaining NIR images instead of using images in VW systems.Different iris characteristics can be evaluated for future work, which also help boost system preciseness and can be checked on other databases to enhance the system activity in real-time applications. This work has been extracted in a mutual iris database. It must be used in non-cooperative databases to see how it functions in such databases. Other problems include shifting iris and distant iris. These databases only contain static images from the eye and less than $50 \mathrm{~m}$ from the camera to the iris. These variables must also be dealt with in the near future.

\section{CONFLICT OF INTEREST}

The authors have no conflict of relevant interest to this article.

\section{REFERENCES}

[1] M. Choudhary, V. Tiwari, and U. Venkanna, "Enhancing human iris recognition performance in unconstrained environment using ensemble of convolutional and residual deep neural network models," Soft Comput., vol. 24, no. 15, pp. 11477-11491, 2020, doi: 10.1007/s00500-019-04610-2.

[2] R. F. Soliman, M. Amin, and F. E. Abd El-Samie, "Cancelable Iris recognition system based on comb filter," Multimed. Tools Appl., vol. 79, no. 3-4, pp. 2521-2541, 2020, doi: 10.1007/s11042-019-08163-2.

[3] M. Omran and E. N. AlShemmary, "Towards accurate pupil detection based on morphology and Hough transform," Baghdad Sci. J., vol. 17, no. 2, pp. 583-590, 2020, doi: 10.21123/bsj.2020.17.2.0583.

[4] A. A. Nithya and C. Lakshmi, "Enhancing iris recognition framework using feature selection and BPNN," Cluster Comput., vol. 22, pp. 12363-12372, 2019, doi: 10.1007/s10586-017-1619-4.

[5] H. M. Ahmed and M. A. Taha, "A Brief Survey on Modern Iris Feature Extraction Methods," vol. 38, no. 1, pp. 1-7, 2020.

[6] A. Ramola, A. K. Shakya, and D. Van Pham, "Study of statistical methods for texture analysis and their modern evolutions," Eng. Reports, vol. 2, no. 4, pp. 1-24, 2020, doi: 10.1002/eng2.12149.

[7] S. A. Alazawi, N. M. Shati, and A. H. Abbas, "Texture features extraction based on GLCM for face retrieval system," Period. Eng. Nat. Sci., vol. 7, no. 3, pp. 14591467, 2019, doi: 10.21533/pen.v7i3.787.

[8] G. Samara and T. Alhmiedat, "Performance Analysis of Dynamic Source Routing Protocol," no. 3, pp. 814-825, 2017.

[9] A. Badr and A. Abdul-Hassan, "A Review on Voicebased Interface for Human-Robot Interaction," Iraqi J. Electr. Electron. Eng., vol. 16, no. 2, pp. 1-12, 2020, doi: 10.37917/ijeee.16.2.10.

[10] H. Proença and L. A. Alexandre, "UBIRIS: A noisy iris image database," Lect. Notes Comput. Sci. (including Subser. Lect. Notes Artif. Intell. Lect. Notes Bioinformatics), vol. 3617 LNCS, pp. 970-977, 2005, doi: 10.1007/11553595_119.

[11] M. N. Othman, "Fusion techniques for iris recognition in degraded sequences," no. March, pp. 4-5, 2016.

[12] T. Kazakov, "by A thesis submitted to University of Birmingham School of Computer Science University of Birmingham," no. May, 2014.

[13] M. A. Taha, "Improved Mesh _ Based Image Morphing," no. 5, 2017.

[14] K. Devi, P. Gupta, D. Grover, and A. Dhindsa, "An effective texture feature extraction approach for iris recognition system," Proc. - 2016 Int. Conf. Adv. Comput. Commun. Autom. (Fall), ICACCA 2016, pp. 1-5, 2016, doi: 10.1109/ICACCAF.2016.7749001.

[15] A. Kumar, A. Potnis, and A. Singh, "Iris recognition and feature extraction in iris recognition system by employing 2D DCT," IRJET Int. Res. Comput. Sci. Softw. Eng. Technol., vol. 3, no. 12, pp. 503-510, 2016.

[16] P. Samant, "Analysis of computational techniques for diabetes diagnosis using the combination of iris-based features and physiological parameters," Neural Comput. Appl., vol. 9, 2019, doi: 10.1007/s00521-019-04551-9.

[17] Q. Luo, Y. Wang, Y. Zheng, X. Chang, B. Wang, and R. Zeng, "High-dimensional co-occurrence matrix: A new tool for 3D seismic target visualization and interpretation," Interpretation, vol. 8, no. 4, pp. 967-979, 2020, doi: 10.1190/int-2019-0127.1.

[18] J. Daugman, "How Iris Recognition Works," Essent. Guid. to Image Process., vol. 14, no. 1, pp. 715-739, 2009, doi: 10.1016/B978-0-12-374457-9.00025-1.

[19] S. Swati and D. Gupta Deepak, "Iris Recognition Using Gabor," Int. J. Comput. Technol. Appl., vol. 4, no. 1, pp. $1-7,2013$.

[20] E. Rydgren, T. Ea, F. Amiel, F. Rossant, A. Amara, and I. Fea-, "IRIS FEATURES EXTRACTION USING WAVELET PACKETS Erik Rydgren, Thomas Ea , Frédéric Amiel , Florence Rossant, Amara Amara To cite this version : HAL Id : hal-02882950," 2020.

[21] A. Azizi and H. R. Pourreza, "Efficient IRIS Recognition through Improvement of Feature Extraction and subset Selection," no. June, 2009, [Online]. Available: http://arxiv.org/abs/0906.4789.

[22] T. W. Ng, T. L. Tay, and S. W. Khor, "Iris recognition using rapid Haar wavelet decomposition," ICSPS 2010 - 
Proc. 2010 2nd Int. Conf. Signal Process. Syst., vol. 1, pp. 820-823, 2010, doi: 10.1109/ICSPS.2010.5555246.

[23] C. Te Chu and C. H. Chen, "High performance iris recognition based on LDA and LPCC," Proc. - Int. Conf. Tools with Artif. Intell. ICTAI, vol. 2005, no. 3, pp. 417421, 2005, doi: 10.1109/ICTAI.2005.71.

[24] V. Velisavljevic, "Low-complexity iris coding and recognition based on directionlets," IEEE Trans. Inf. Forensics Secur., vol. 4, no. 3, pp. 410-417, 2009, doi: 10.1109/TIFS.2009.2024025.

[25] Rashad, Shams, Nomir, and El Awady, "IRIS Recognition Based On LBP and Combined LVQ Classifier," Int. J. Comput. Sci. Inf. Technol., vol. 3, no. 5, pp. 67-78, 2011, doi: 10.5121/ijcsit.2011.3506. 